
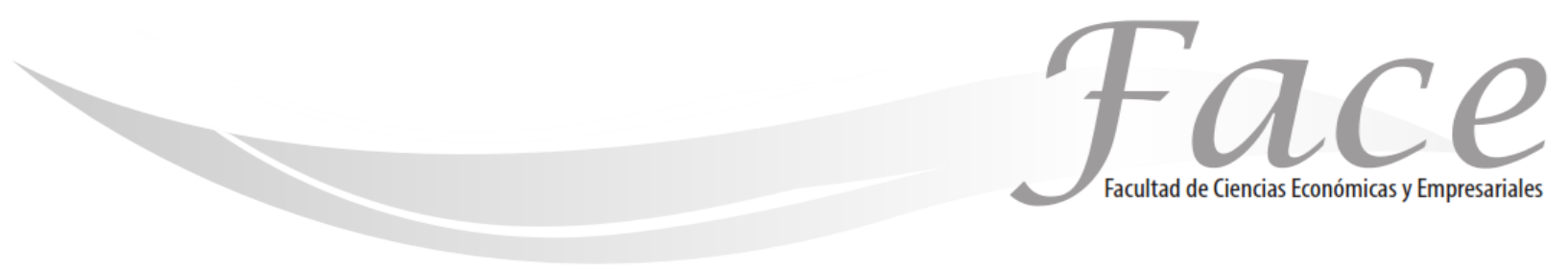

ISSN Impreso: 1794-9920

ISSN Electrónico: 2500-9338

Volumen $19-\mathrm{N}^{\circ} 2$

Año 2019

Págs. $41-50$

(c) (1) $\Theta \Theta$

\title{
ESTRATEGIA DELIBERADA Y EMERGENTE EN EMPRENDIMIENTO: PROPUESTAS DESDE UN ENFOQUE COGNITIVO
}

\author{
Juan Morúa Ramírez \\ ORCID: https://orcid.org/0000-0001-5080-5243 \\ Mariela Montoya Sanchez \\ Kenya Gabriela Aguirre Bustos
}

Fecha de Recepción: Junio 15 de 2019

Fecha de Aprobación: Octubre 7 de 2019

\section{Resumen:}

Proponer desde un enfoque cognitivo una ayuda visual para pilotear la estrategia deliberada de los emprendedores y establecer una guía de vigilancia estratégica para la creación de estrategias emergentes basada en la dinámica de los sistemas. Se utiliza una posición epistemológica constructivista y se basa en un método de intervención acción. Se establecen una ayuda visual basadas en el flujo de efectivo para pilotear la estrategia deliberada y se establece a la variabilidad, las restricciones y la incertidumbre como elementos para la reflexión en la creación de estrategias emergentes. Los emprendedores se deben de basar en la heurística para establecer estrategias tanto deliberadas como emergentes.

Palabras clave: Estrategia, emprendimiento, cognición.

\footnotetext{
* Doctor en Gestión. Universidad de Guanajuato, División de Ciencias Sociales y Administrativas. Contacto: juan.morua@ugto.mx.

* * Estudiante de la Licenciatura en Administración Financiera, Universidad de Guanajuato, División de Ciencias Sociales y Administrativas. Contacto: mari_elal1a@hotmail.com

* * * Estudiante de la Licenciatura en Administración Financiera, Universidad de Guanajuato, División de Ciencias Sociales y Administrativas. Contacto: kenyaaguirre@hotmail.com
} 


\title{
DELIBERATE AND EMERGING STRATEGY IN ENTREPRENEURSHIP: PROPOSALS FROM A COGNITIVE APPROACH
}

\begin{abstract}
:
Propose from a cognitive approach a visual aid to pilot the deliberate strategy of entrepreneurs and establish a strategic surveillance for the creation of emergent strategies based on the dynamics of the systems. A constructivist epistemological position is used and is based on a method of action intervention. A visual aid based on cash flow is established to pilot the deliberate strategy and a proposition to use variability, restrictions and uncertainty as elements for reflection in the creation of emerging strategies. Entrepreneurs should be based on heuristics to establish strategies deliberate and emergent.
\end{abstract}

Keywords: Strategy, entrepreneurship, cognition

\section{ESTRATÉGIA DELIBERADA E EMERGENTE NO EMPREENDEDORISMO: PROPOSTAS DE UMA ABORDAGEM COGNITIVA}

\section{Resumo:}

Propor, a partir de uma abordagem cognitiva, um auxílio visual para pilotar a estratégia deliberada dos empreendedores e estabelecer uma vigilância estratégica para a criação de estratégias emergentes baseadas na dinâmica dos sistemas. Uma posição epistemológica construtivista é usada e é baseada em um método de intervenção de ação. Um auxílio visual baseado no fluxo de caixa é estabelecido para testar a estratégia deliberada e uma proposição para usar a variabilidade, as restrições e a incerteza como elementos de reflexão na criação de estratégias emergentes. Os empresários devem se basear em heurísticas para estabelecer estratégias deliberadas e emergentes.

Palavras-chave: Estratégia, empreendedorismo, cognição. 


\section{INTRODUCCIÓN:}

El emprendimiento tiene características particulares, sobre todo si el individuo ya se encuentra en la acción, es decir desarrollando su proyecto de emprendimiento. Entre las características que más resaltan comúnmente se encuentran: la falta de experiencia del emprendedor y la falta de efectivo. Aun así, de manera formal o informal el emprendedor establece estrategias que definen los objetivos a largo plazo y la manera de operar su proyecto para alcanzar dichos objetivos.

Es de recalcar que según nuestras observaciones y experiencia en el campo derivadas de trabajos precedentes (Morua, 2013; Morua, 2017) los emprendedores se enfrentan a un mundo dinámico, en donde emergen nuevas características, por ejemplo: nuevas leyes 0 reglamentos, nuevas preferencias 0 tendencias de mercado, nuevas formas de competir, etc. Ante esta falta de experiencia y de recursos monetarios escasos que enfrentan los emprendedores es necesario establecer estrategias deliberadas, es decir: derivadas de planes, establecidas en el proyecto emprendedor o por modelos 0 patrones de comportamiento. Estas estrategias requieren de apoyos visuales para medir el avance 0 las desviaciones de lo planeado.

Por otro lado, la dinámica de la organización y del medio ambiente, requiere que el emprendedor y su equipo aprendan a identificar cambios en el contexto o que permiten crear una estrategia emergente, esto resalta la necesidad de establecer mecanismos de vigilancia estratégica hacia ciertos elementos que afecten el desarrollo de su estrategia deliberada creando con esto estrategias emergentes. En efecto, la estrategia emergente surge por oportunidades o amenazas detectadas del medio ambiente y del contexto. En este sentido surge la necesidad de aprender a detectar ciertos fenómenos ligados a la dinámica de los sistemas.

El presente artículo está basado en una posición constructivista, enfocado hacia el individuo y su aprendizaje, nuestro supuesto es que la estrategia del emprendedor debe ser conducida como un proceso de experimentación por lo que deben de ser portadores de heurística. Para esto el presente artículo esta dividido en tres secciones: en la primera justificamos la posición epistemológica a seguir, el constructivismo, y establecemos el método, intervención acción. La segunda parte expone el marco teórico en donde se tratan a la estrategia deliberada y a la estrategia emergente, en un segundo punto se estudian los limitantes del desempeño de un sistema basados en su dinámica y en un tercer punto se describe el proceso cognitivo que siguen los
emprendedores. Finalmente, en la tercera parte exponemos los resultados en donde se propone, basados en las teorías cognitivas, una guía visual que permita pilotear la estrategia deliberada utilizando al flujo de efectivo como elemento conductor, y para la identificación de estrategias emergentes proponemos la reflexión sobre los elementos limitantes de los sistemas dinámicos.

\section{MARCO TEÓRICO:}

\section{Emprendimiento entre estrategia deliberada y estrategia emergente}

Henry Mintzberg y James A. Waters (1985) establecen dos tipos ideales de estrategia: la deliberada y la emergente. Entienden que las estrategias deliberadas y emergentes deben concebirse como dos finales/polos/extremos de un continuo a través del cual discurren las estrategias del mundo real. Es decir, que hay toda una serie de tipos de estrategia que se mueven por ese continuo. En este sentido, Mintzberg y Waters (1985) difícilmente podemos encontrar estrategias perfectamente deliberadas 0 perfectamente emergentes en las organizaciones. En efecto, la dinámica tanto de los mercados como de las organizaciones hace difícil una estabilidad suficiente para que una estrategia deliberada pueda ser llevada a cabo. En el caso de las estrategias emergentes, estas requieren de un número suficiente de expertos, información y vigilancia estratégica que consumiría muchos recursos de la organización y aun así no permitirían analizar todos los elementos del contexto.

Así, según Mintzberg (1987) por un lado se encuentran las estrategias deliberadas son aquellas en las que las intenciones existían antes de que fueran realizadas, es decir que fueron creadas por un plan, se estableció un modelo estratégico a seguir o surgieron por la continuidad de acciones que en el futuro sirvieron para alcanzar los objetivos. Por otro lado, surgen las estrategias emergentes, que se desarrollan en ausencia de planes, modelos 0 a pesar de ellos (subyacentes, no percibidos). Esto surge por las condiciones detectadas del contexto. En el caso del emprendedor, es de notar que todo proyecto está inscrito en el futuro y por tanto el emprendedor debe de ser capaz de proyectarse en él (Schmitt, 2015), identificar posibles 
problemas que limiten su acción empresarial y anticiparse a la aparición de estos. Es decir, poder detectar cambios emergentes resulta útil realizar ejercicios de problematización, establecer los posibles limitantes y restricciones, identificándolos en los diferentes periodos de tiempo futuro, que pueden afectar el alcance de los objetivos.

Otro ejercicio complementario en la emergencia es la anticipación, la cual marca la necesidad de actuar hoy para que los posibles problemas del futuro aparezcan. Es en este sentido que problematización y anticipación tienen el carácter estratégico. Dicho lo anterior se plantea que en un proyecto emprendedor resulta conveniente hacer uso de estrategias deliberadas monitoreadas por medio de herramientas de ayuda visual que nos dan detalles de la situación en determinado tiempo, que se convertirían en común denominador para los actores de la organización al interpretar dicha ayuda visual.

Es de remarcar que la dinámica que presenta cada proyecto emprendedor impide trabajar con base en puras estrategias deliberadas por lo que los individuos deben hacer uso de las estrategias emergentes, ya que resulta improbable que un proyecto emprendedor se desarrolle en un ambiente en el que alguno de sus elementos no sea interferido por una fuerza externa, o que se encuentre en un entorno predecible con un control total llegando a una situación ideal.

\section{Limitantes del desempeño según la dinámica de sistemas}

Una forma de evaluar la estrategia es a través de la evaluación del desempeño. Siguiendo la figura 1 . Se observa que la base del desempeño parte de la pertinencia entre los recursos y los objetivos determinados y usados por el emprendedor. Posteriormente, utilizando la eficacia, relación entre objetivos y resultados y, finalmente se utiliza a la eficiencia, que define una razón entre los resultados obtenidos y los recursos alcanzados.

Figura 1.

EL DESEMPEÑO

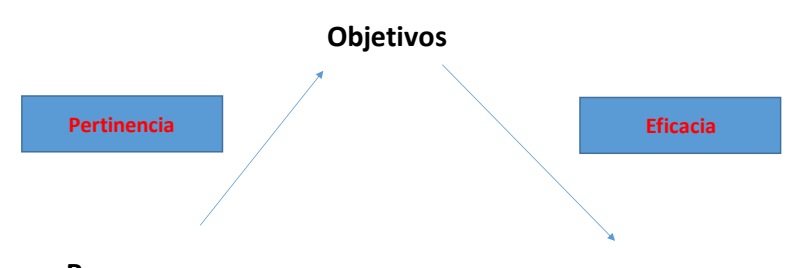

Recursos

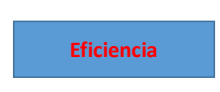

Resultados

Fuente: Elaboración Propia

Pero recordemos que la evaluación del desempeño de la estrategia es ex -post, el emprendedor requiere de desarrollar sus capacidades de problematización, es decir establecer escenarios que dicten dificultades futuras, y de anticipación, es decir realizar acciones para que los escenarios pesimistas no se cumplan. En otras palabras, utilizar un mecanismo que le ayude a construir estrategias emergentes. La problematización y anticipación deben de tener una guía que permita la reflexión estratégica. Para elaborar dicha guía nuestra propuesta se basa en considerar los limitantes del desempeño de los sistemas organizacionales: variabilidad, restricción e incertidumbre (Morua, 2013). Dichos limitantes están basados en la dinámica de los sistemas.

\section{Variabilidad}

La variedad y la variabilidad por sí mismas, son fenómenos característicos del comportamiento de los sistemas. En efecto, los sistemas en su desarrollo y evolución tienden a modificar la variedad de sus subsistemas, dicha variedad con el paso del tiempo y para un observador se convierte en variabilidad, la cual estudiará mediante la estadística comparando los valores obtenidos con una medida de tendencia central. Es de notar que a mayor dinámica organizacional mayores posibilidades de cambios, es decir mayor variabilidad. Es de notar que los sistemas algunas veces no requieren de intervención en el control de su variabilidad, siempre y cuando esta se encuentre en los límites operacionales del sistema. Es de remarcar que en programas como los de mejora continua, en el caso de calidad, los límites de control son cada vez más estrechos, por ejemplo: en las herramientas seis sigma. En este caso la variabilidad está ligada al concepto de control. Todos aquellos sistemas que tengan valores fuera de los aceptados deben de ser estudiados y controlados para volverlos a valores dentro de los límites.

\section{Restricción}

En el caso de la variedad de valores que puede adoptar un sistema y de su variabilidad, hemos hablado de un sistema como ente aislado, pero debemos remarcar que existe un fenómeno llamado covarianza. La covarianza es la relación entre la varianza de diferentes sistemas en interrelación. Así, la varianza de un sistema puede afectar el desempeño de otros. Este fenómeno es llamado restricción, que por definición es cuando un elemento limita el desempeño de uno o varios componentes. Estableciendo entonces que entre más elementos se encuentren en interacción y más heterogéneos sean estos, será más evidente la aparición de restricciones. En el caso de los proyectos emprendedores estos están llenos de elementos heterogéneos, así como integrados por una red de actores. Esta red, como marca la teoría de sistemas, por un lado, favorece al proyecto, dado que el todo es más grande que la suma de sus partes, pero de manera paradójica puede hacer que existan elementos que limiten su desempeño.

Esta paradoja está reflejada en la teoría de la estructuración de A. Giddens (2005) que marca que toda 
estructura organizativa tiende a habilitar, es decir a permitir y asegurar, la acción, pero a su vez esta misma restringe la acción misma. Así mismo, se establece en la teoría de la complejidad de E. Morin (2005), el cual en su principio hologramático marca que el todo y las partes también se limitan entre sí mismas, estableciendo también una cierta paradoja ontológica entre todo y partes. Con lo anteriormente explicado, parece evidente que dentro de la elaboración de una estrategia emergente se analice la aparición de dichas restricciones con mira a su eliminación o hacia la reducción de sus efectos.

\section{Incertidumbre}

El último elemento por considerar tiene que ver con la información y la relación con el individuo, no solamente en términos de su capacidad para procesar, codificar y actuar en base a esta, también con respecto a sus características psicológicas y su relación con las hipótesis elaboradas para la acción que utiliza gracias a la información. Esto resalta en importancia si recordamos que los proyectos emprendedores están inscritos en el futuro, en donde no se tiene información exacta de los sucesos y de las acciones. Esta situación hace que crezca la importancia de la estrategia emergente, en donde nos tenemos que preparar estableciendo escenarios construidos en base a una pobre información. Esto afirma la sentencia de E. Morin (2005): la incertidumbre llama a la estrategia.

Las fuentes de incertidumbre son claras, por un lado, tenemos los limitantes cognitivos, de memoria y capacidad de procesamiento cerebral de los individuos y, por el otro lado, tenemos sus características psicológicas frente a la toma de decisiones con poca información, por ejemplo: un individuo con información suficiente puede dudar en su actuar 0 en su reflexión por causa de la incertidumbre 0 al contrario, un individuo con poca información pude lanzarse en un proyecto (Morua y Gómez, 2016).

La dinámica de las organizaciones y del medio ambiente incrementa la necesidad de establecer estrategias emergentes. Por ejemplo: se observa actualmente una mayor volatilidad en las preferencias de un cliente 0 en el aumento de leyes y reglamentos que norman el actuar de las empresas.

Pese que actualmente la información fluye de manera más rápida, esta requiere de un tiempo considerable en su procesamiento, pero recordemos que también el tiempo es un recurso escaso. Así el emprendedor debe de ser capaz de identificar que variables debe de vigilar, es decir que información obtener del medio ambiente para poder tomar decisiones estratégicas.

\section{El emprendedor y los procesos cognitivos}

Anteriormente mencionábamos que en el presente artículo seguiríamos una posición epistemológica constructivista. Así nos enfocaremos también en el individuo y su cognición, es decir, en la manera en como conoce. En el constructivismo, el conocer está relacionado íntimamente con la acción del individuo, específicamente con la interacción de sus sentidos. La cognición entonces depende tanto de procesos reflexivos e interpretativos que realiza el individuo como de su acción. De hecho, Varela y Maturana (Proulx, 2008) le dan tanto peso a esta relación que acuñan el concepto de enacción, en donde se especifica que no existe una sola definición de lo real, sino que existe una interpretación subjetiva de la realidad que depende no solamente de los procesos cerebrales del individuo y de su memoria sino de la acción de sus sentidos.

Ciertamente en condiciones estables es relativamente fácil la construcción de conocimientos, se crean expertos que pueden predecir, controlar y establecer de manera determinista los resultados de los fenómenos (Morua y Goméz 2016), pero frente a sistemas dinámicos con interacción de elementos heterogéneos, como en el caso del emprendimiento, la cognición no es tan sencilla, dado que la creación de representaciones, los objetos y sus fenómenos tienden a aparecer ante la conciencia del individuo como constitutivos de diferentes esferas de su realidad (Berger y Luckmann, 2001). Así, el emprendedor tiende a dar importancia relativa, banal o fugaz a los objetos, pausando, retomando o ignorando las reflexiones sobre los mismos.

Además, recordemos que el emprendedor está involucrado en proyectos que se inscriben en el futuro, el cual no está predeterminado, sino que se encuentra por construir. Así, su labor es más complicada dado que solamente cuenta con hipótesis plausibles sobre los fenómenos que aún no se presentan y quizá nunca lo hagan, bajo este sentido podemos entonces establecer que el futuro se construye paso a paso y no en una lógica adivinatoria 0 de trasposición del pasado hacia al futuro (Schmitt, 2015).

Es entonces donde el individuo recurre, por ejemplo, a la memoria, la experiencia o la teoría para proyectarse construyendo y rechazando hipótesis conforme a la manera en que su percepción sobre el medio ambiente y sus representaciones creadas le vayan indicando. Pero recordemos que el emprendedor aun no cuenta con experiencia previa y muchas veces cuenta con una teoría pobre sobre temas administrativos y algunas veces inclusive sobre cuestiones técnicas de su proyecto.

Por el lado de la acción, que está ligada a la cognición, es de notar que estas tienen el carácter de contextual, es decir que aunque el individuo haya realizado un proceso reflexión-hipótesis, el contexto en el que se encuentra puede hacer que tanto las acciones como las intenciones cambien, por lo que la acción solamente puede ser calificada una vez terminada ésta y el individuo puede 
justificar su acción mediante un nuevo proceso de reflexión (Kechidi, 2005). A su vez, es importante recalcar que el individuo no es un ser aislado, el proceso reflexivo requiere además de cierto esfuerzo lógico, memoria y experiencia, necesita información. Dicha información se obtiene del medio ambiente y es derivada de su interacción con este. Bajo este sentido el emprendedor como individuo interactúa con otros actores los cuales también declaran y dan existencia a objetos mediante su subjetividad (Berger y Luckmann, 2001), Esto resalta la importancia de crear medios de comunicación, reflexión e interpretación, como el lenguaje (Piaget, 1954) y la construcción de modelos (Le Moigne, 1999) para poder interactuar y explicitar sus ideas, intenciones y proyectos con el grupo (Morua, Estrada y Quintero, 2015).

Así, resulta pertinente el establecer estrategias para anticipar la posible aparición de limitantes. Esto implica actuar, reflexionar y elaborar hipótesis partiendo de una intención coherente. En otras palabras, en lugar de certeza, el emprendedor debe de aprender a construir coherencia y sentido en su proyecto emprendedor en la acción misma (Schmitt, 2015).

\section{METODOLOGIA. investigación-acción:}

Nuestro trabajo es continuidad de trabajos derivados de una investigación-acción, es decir de observaciones previas en el campo (Morua, 2017). Recordemos que en el constructivismo se sigue un bucle entre la literatura, teorías, y el estudio del campo, práctica. El propósito de investigación-acción es principalmente de involucramiento y mejora. Por un lado, el involucramiento se refiere la participación del sujeto investigador en el proceso en todas las fases de planeación, acción, observación y reflexión. Por otro lado, la mejora es la manera cómo la intervención social particular cambia la situación, intensificando el entendimiento de los sujetos investigados en su habilidad para controlar la situación y rehacer la práctica de mejora (Schwandt, 2007).

En el estudio previo llevado a cabo con emprendedores se observó la necesidad de construir guías estratégicas generales que permitan la reflexión, no en el sentido de analizar fortalezas, debilidades, oportunidades y amenazas, sino en un sentido inspirado en la dinámica de sistemas. El objetivo es ofrecer conocimientos que puedan ser llevados a la acción, estableciendo conceptos generales que sirvan como base para construir estrategias para la acción. De este modo se realizó una revisión bibliográfica extensiva sobre:

a) la dinámica de las organizaciones, la teoría de la estructuración, las teorías de la acción y la teoría de la complejidad, para comprender los limitantes del desempeño de las organizaciones desde el punto de vista de la dinámica de sistemas.

b) las ciencias cognitivas y la psicología social, para comprender los procesos de pensamiento y la toma de decisiones

c) El emprendimiento orientado hacia el individuo como constructor de ideas y como ente perteneciente a una red de actores.

Esta revisión nos permitió hace dialogar diferentes ideas de diferentes autores y establecer un marco teórico sólido para comprender los fenómenos y situaciones encontradas en el campo.

\subsection{Material y Método}

Uno de los elementos olvidados en las investigaciones económico-administrativas es la definición de la posición epistemológica de los investigadores, esta resalta en importancia dado que: clarifica la manera en cómo los investigadores definen la realidad y establece la metodología a seguir para validar los conocimientos obtenidos de su investigación. En nuestro caso vamos a utilizar el constructivismo que parte de una lógica de abducción la cual consiste en elaborar una observación empírica ligada a una regla general que tiene una consecuencia, es decir que permita de encontrar la consecuencia si la regla general es verdadera. Según David (2012) existen tres niveles de abducción:

I. Abducción casi-automática, la regla de interpretación es inmediatamente reconocida por el investigador

II. No automática, implica la elección por un observador. La regla debe de ser seleccionada como la más plausible

III. No se trata de seleccionar una regla, sino de inventar reglas nuevas que permitan la inferencia, creatividad y cambio de paradigma.

Así, los razonamientos científicos combinan los tres tipos de lógica (figura 2): la abducción, la deducción y la inducción, bajo el siguiente esquema:

- Una hipótesis explicativa es construida por abducción

- Las consecuencias posibles a esta hipótesis son exploradas por la deducción

- La inducción permite una actualización de las reglas o teorías utilizadas

- Si las reglas son invalidadas se requiere reformular la hipótesis 
Figura 2.

Relación Entre la Lógica De Abducción, Deducción e

Inducción

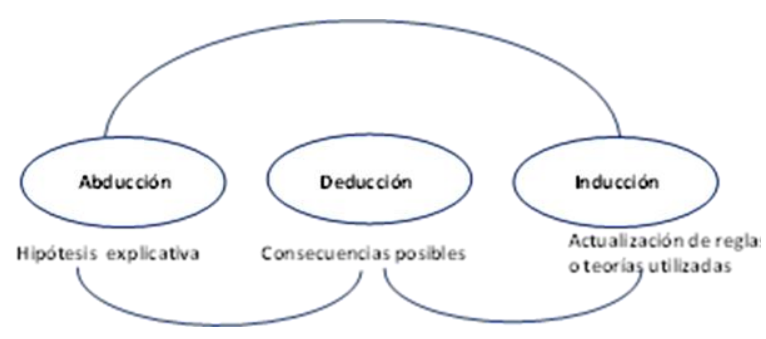

Fuente: Elaboración Propia

El constructivismo también parte de la idea de la no separación entre el objeto y el sujeto, dándole más peso a este último, estableciendo que el aprendizaje y la cognición solamente proviene del individuo y la relación de sus sentidos con el objeto. Entendiendo al constructivismo desde la diferencia entre saber y conocimiento, este último descrito visto como un proceso que se desarrolla por $y$ para la experiencia, cuyo objetivo es el de elaborar cuadros mentales viables y funcionales que se encuentran en el interior de los sujetos (Parmentier y Avenier, 2013, mencionado por Morua y Larios-Gómez, 2017).

En este caso, la información adquirida del medio ambiente que no ha sido puesta en práctica tiene el carácter de hipótesis plausible. Así, los individuos mantienen una realidad interpretada. Es decir, desarrollan un significado subjetivo construyendo un mundo coherente alrededor de ellos (Berger y Luckmann, 2001).

En este caso, las investigaciones basadas en el constructivismo establecen solamente hipótesis plausibles o supuestos los cuales para ser comprobados deben de ser llevados a la acción por individuos, los cuales desarrollarán un proceso cognitivo con dichos postulados. En otras palabras, los resultados generados deben de ser orientados hacia la acción (Avenier, 2004).

En un sentido clásico de la ciencia, este proceso causa polémica dado que los investigadores construyen un marco teórico jamás completo, pero que puede ser llevado a la acción, e inician sus investigaciones bajo una lógica abductiva en lugar de una deductiva o una inductiva, como lo marca la posición de la ciencia dominante (David,1999).

\section{RESULTADOS:}

La estrategia deliberada y la representación visual del flujo del efectivo.

Es de reconocer que los emprendedores, al menos en México, se enfrentan a varios problemas de distintos ámbitos, tales como legales, ambientales, económicos, financieros. Con respecto a este último podemos poner énfasis al problema del flujo de efectivo. Esto parece lógico si consideramos que cuando un emprendedor pone en marcha su idea de negocio necesita de varios recursos, especialmente los recursos monetarios, teniendo varias fuentes de financiamiento, entre ellas: dinero propio, proveedores 0 algún préstamo. Para lograr un capital propio considerable para la puesta en marcha de tal negocio, el emprendedor generalmente destina una gran parte de sus ahorros para lograrlo.

Es por esta razón que cuando el negocio inicia y va en desarrollo, no se tienen los ingresos esperados, se presentan problemas en el flujo de efectivo, sumando además los errores en la toma de decisiones estratégicas, dado que el emprendedor se encuentra en un proceso de aprendizaje, y en la pobre utilización de las palancas financieras, debido a la falta de confianza de los bancos.

Por estas razones un mecanismo de vigilancia de la estrategia deliberada es el establecimiento de un cuadro de mando orientado hacia el flujo de efectivo para los emprendedores. Dicho cuadro se presenta en la figura 3, la cual está compuesta de diferentes contenedores de efectivo: acreedores, caja y bancos, clientes, almacén de productos terminados (APT), proveedores, producción, gastos generales y finalmente utilidad. 
Figura 3.

REPRESENTACIÓN DEL FLUJO DE EFECTIVO

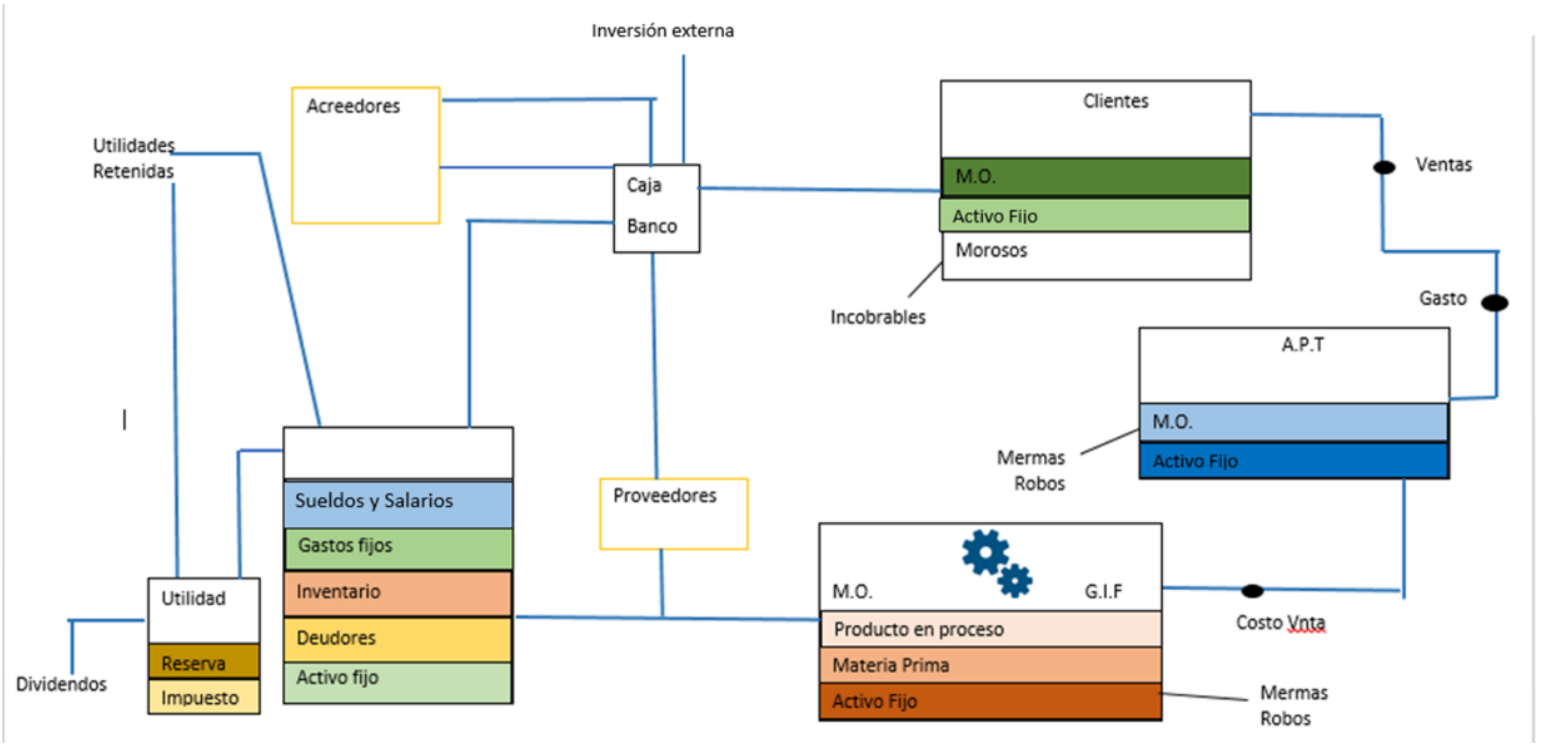

Fuente: Elaboración propia

Cada contenedor cambia con respecto al tiempo, guardando una cierta cantidad de efectivo, ya sea en forma de dinero líquido, como en el caso de caja y banco, de dinero en forma de mercancías, como en el caso de producción y APT, en forma de cuentas por cobrar, como en el caso de clientes, en forma de gastos, como el contenedor de gastos generales 0 en forma de apalancamiento, como en el caso de proveedores y acreedores. Es de notar que dicha representación no sigue de manera formal la idea contable de la separación de activos, pasivos y capitales. Más bien se establece como una orientación de donde se encuentra el efectivo. Por ejemplo, observemos que el activo fijo se encuentra como parte de los contenedores bajo la idea de que representa dinero que está invertido, si bien dicha inversión ayuda a la generación de más efectivo, finalmente no representa la idea de liquidez de efectivo.

Así, el objetivo es visualizar el estado del efectivo, como pasa de contenedor en contenedor hasta llegar a las utilidades brutas. Dicho contenedor puede adaptarse a los sistemas de control administrativo 0 puede ser representado gráficamente en forma manual, inclusive se puedan anexar parámetros financieros que tienen relación con el efectivo.
Es de notar que para el control financiero se requieren otras medidas financieras, pero el objetivo es la visualización, de esta forma el individuo se puede ayudar a crear representaciones mentales, ayudándolo a estimar la efectividad de su estrategia deliberada y proponer cambios, en el caso de que los contenedores crezcan, existan fugas de efectivo, como en el caso de mermas y robos, o el flujo de efectivo se estanque 0 tenga poca velocidad. Obviamente, esta debe de adaptarse a las especificidades que presenta cada nuevo proyecto en marcha, en cuanto a sus plazos y tiempos.

\section{Los limitantes del desempeño como guía para las estrategias emergentes}

Anteriormente analizamos los limitantes del desempeño de un sistema vistos desde su dinámica. Los tres fenómenos: Variabilidad, restricción e incertidumbre, son omnipresentes dentro de las organizaciones. En efecto, aunque busquemos reducir la variabilidad, por ejemplo: en un programa de control de calidad, la mejora tiende a aproximar más los datos ajustando los límites de control. También es de notar que la variabilidad proviene de la variedad, así cada vez que ofrecemos un nuevo producto 0 servicio o que agregamos una unidad en los procesos de la organización se amplía la necesidad de control. Esto en función de la ley de la variedad requerida establecida por Asbhy (Boisot \& McKelvey, 2011). 
El caso de la restricción es la misma, la integración de un nuevo elemento a la red de trabajo agrega una nueva posibilidad de aparición de una restricción. Anteriormente mencionábamos la paradoja del trabajo en equipo, que si bien el todo (equipo) habilita a cada una de sus partes, este también tiende a limitarlas. Eliminar una restricción hace que aparezca otra, de ahí la necesidad de establecer un proceso continuo de identificación y eliminación de restricciones.

La incertidumbre también es omnipresente, la información fluye de manera continua, pero hemos estudiado que se requeriría una gran cantidad de recursos y tiempo para analizarla, recordemos que el emprendedor tiene limitados ambos elementos. También, la incertidumbre tiene que ver con las características psicológicas del individuo, si bien la experiencia puede ayudar a la toma de decisiones en casos de amplia incertidumbre, recordemos que el emprendedor regularmente se encuentra en fase de aprendizaje.

Es importante mencionar que estos tres limitantes se retroalimentan los unos con los otros, es decir: a mayor variabilidad, mayor incertidumbre, a mayor cantidad de relaciones, mayor incertidumbre, a mayor restricción mayor variabilidad, a mayor restricción mayor incertidumbre. En base a estos tres fenómenos: Variabilidad, restricción e incertidumbre se pueden establecer programas con equipos de trabajo multidisciplinarios que tengan como finalidad problematizar, es decir detectar la aparición de posibles problemas que afecten los objetivos de la organización, ejercicio que deriva en el establecimiento de estrategias emergentes.

El resultado de la problematización debe de ser un plan estratégico, que tenga el carácter de emergente. Esto hace que el ejercicio de reflexión/hipótesis/acción sea necesario e involucre también un ejercicio de anticipación de elementos no deseados, los que definimos como la construcción de escenarios y la puesta en marcha de acciones para evitar que los limitantes sucedan.

El ejercicio de problematización y anticipación debe de ser sistemático, orientado a motivar la reflexión de los individuos, al establecimiento de escenarios y a la creación de hipótesis, a la anticipación y a la acción. Por lo que se sugiere que se utilicen herramientas heurísticas, las cuales motivan a la acción creativa y que se realicen en equipos interdisciplinarios.

\section{CONCLUSIONES:}

Uno de los elementos que nos ayudan a reflexionar y construir representaciones sobre los problemas que enfrentamos es la construcción de modelos (Le Moigne, 1994). Estos representan una ayuda visual e involucran un ejercicio cognitivo. Como hemos establecido la estrategia deliberada que crea un emprendedor para llevar a cabo su proyecto requiere de una ayuda visual para evaluarla, pilotearla e inclusive para corregirla.

Nuestra propuesta se basa en la representación del flujo de efectivo. La importancia de dicho cuadro radica en el hecho que para el emprendedor el efectivo representa regularmente un recurso escaso. Por lo que las acciones afectan de una u otra forma al flujo. Nuestra intención no es realizar una representación contable, más bien una ayuda visual para conocer el estado del efectivo como guía para la estrategia. Es importante destacar que la propuesta se ha estado perfeccionando desde trabajos anteriores (Morua, 2013) y hasta llegar a la propuesta que presentamos en este artículo, la cual pondremos en marcha con una serie de emprendedores en el corto plazo.

Por otro lado, debemos de hacer notar que el dinamismo es una de las características menos consideradas en gestión (Morua, 2015) aunque existan numerosos estudios que lo mencionan, regularmente no se tratan a fondo sus características ni mucho menos sus componentes. En el caso de emprendedor, aunque este interactúe con sistemas que parecen tener una dinámica simple, hemos notado que la complejidad de los negocios está creciendo y al parecer seguirá haciéndolo.

En este sentido parece pertinente la construcción de guías cognitivas que contemplen los limitantes de la dinámica de un sistema para crear estrategias emergentes. Así, podemos concluir que los emprendedores se deben de basar en procesos heurísticos para la construcción de estrategias para sus proyectos. 


\section{REFERENCIAS:}

Avenier, M.-J. (2004). L'élaboration de savoirs actionnables en PME légitimés dans une conception des sciences de gestion comme des sciences de l'artificiel. Revue international PME, Vol. 17 No. 3-4 p. 13-42.

Berger, P., \& Luckmann, T. (2001). La construcción social de la realidad. Buenos Aires: Amorrurtu .

Boisot, M., \& McKelvey, B. (2011). Complexity and organisation-Environment. Relations: Revisiting Ashby's law of requisite variety. In P. Allen, S. Maguire, \& B. McKelvey, Complexity and Management (pp. 279-298). London: Sage.

David, A. (1999). Logique, épistémologie et méthodologie. Conférence de l'AIMS mai 1999. Paris.

David, A. (2012). La recherche-intervention, cadre général pour la recherche en management? Dans A. David, A. Hatchuel, \& R. Laufer, Les nouvelles fondations de sciences de gestion (pp. 241-264). Paris: Presses de Mines.

Giddens, A. (2005). La constitution de la société. Paris: PUF.

Kechidi, M. (2005). La théorie de la structuration, une analyse des formes et des dyamiques. Relations industrielles/ Industrial relations Vol.60 No. 2, 348 à 369.

Le Moigne, J.-L. (1994). La théorie du système général : théorie de la modélisation. Paris: Presses universitaires de France.

Le Moigne, J.-L. (1999). La modélisation des systèmes complexes. Paris: Dunod.

Mintzberg, H. (1987). The Strategy Concept I: Five Ps for Strategy, California Management Review, $n^{0} 30-3,11$ 24.

Mintzberg, H. y waters, J.A. (1985). Of Strategies, Deliberate and Emergent, Strategic Management Journal, Vol. 6, 257-272

Morin, E. (2005). Introduction à la pensée complexe. Paris: Éditions du seuil.
Morua, J. (2013). Pour une conception stratégique de la valeur des produits et services, une approche dynamique en PME. Nancy: Thèse en sciences de la gestion, Université de lorraine.

Morua, J. (2017). El emprendedor, su proyecto y sus limitantes desde un enfoque dinámico y cognitivo: reflexiones de una intervención en campo. Proyectics, proyectica, proyectique No. 17. 51-67.

Morua, J., \& Gómez, L. (2016). La construction de représentations face au dynamisme des organisations: réflexions pour les sciences de gestion. Dans C. Schmitt, De la complexité de l'action dans les organisations (pp. 71-86). Berne: Growth Publiser.

Morua, J., Estrada, S., \& Quintero, Y. (2015). Emprendimiento, complejidad y representaciones: entre acción, proyección e interacción. Projectics, Proyéctica, Projectique Vol. 3 No.15, 49-71.

Morua, J., \& Larios-Gómez, E. (2017). Capitulo 22 Epistemología y accionabilidad en las ciencias de gestión. En Administración. Un enfoque latinoamericano México-Brasil Coord. Larios-Gómez, E., \& Giuliani, A. C., 2017. ISBN LIBRO IMPRESO: 978-607-32-4000-0. ISBN E-BOOK: 978-607-32-4005-5 Editorial Pearson : México.

Piaget, J. (1954). Le langage et la pensée du point de vue génétique. Acta psychologica. Vol.10 June, 51-60.

Proulx, J. (2008). Some differences between Maturana and Varela's Theory of Cognition and constructivism. Complexity: An international journal of Complexity and Education Vol. 5, 11-26.

Schwandt, T. (2007). The Sage Dictionary of Qualitative Inquiry. London: SAGE Publications. 